\title{
Instant FaSSIF and FeSSIF-Biorelevance Meets Practicality
}

\author{
Julia Elisabeth Boni ${ }^{1,2}$, Rolf Stefan Brickl', Jennifer Dressmann ${ }^{2,3}$, \\ and Martin L. Pfefferle' \\ 'Pharmaceutical Research \& Development, Boehringer Ingelheim Pharma GmbH \& Co. KG, \\ Biberach an der Riss, Germany \\ ${ }^{2}$ Institute of Pharmaceutical Technology, Johann Wolfgang Goethe University, Max-von-Laue \\ Str. 9, 60438 Frankfurt am Main, Germany
}

e-mail:dressman@em.uni-frankfurt.de

\begin{abstract}
The objective of this study was to prepare an "instant" form of FaSSIF and FeSSIF that could be implemented easily and reproducibly in the laboratory setting. Concentrated solutions containing bile salts and lecithin were freeze-dried and further processed into bulk packs, blisters, or tablets, or sealed in powder form under nitrogen in vials. Physical properties as well as solubility and dissolution data for two poorly soluble compounds, danazol and a Boehringer-Ingelheim development compound (BIXX), were compared with data for freshly prepared FaSSIF and FeSSIF and a commercially available "instant" product. After the freeze-dried FaSSIF and FeSSIF media were reconstituted with appropriate buffers, their properties were close to those of freshly prepared media and a commercially available product for reconstitution, SIF Powder. Additionally, the solubility and dissolution profiles of danazol and BIXX in media prepared by all three methods were similar. Reconstitution of freeze-dried "instant" FaSSIF and FeSSIF appears to be an accurate, reproducible, and efficient way of preparing biorelevant dissolution media.
\end{abstract}

\section{INTRODUCTION}

B iorelevant dissolution testing (1) has made it possible to predict, at least on a rank-order basis, in vivo formulation behavior. Although biorelevant dissolution media like FaSSIF and FeSSIF (Fasted- and FedState Simulated Intestinal Fluid) are not a one-to-one copy of human intestinal fluids, they attempt to simulate the most relevant parameters including $\mathrm{pH}$, osmolality, surface tension, and the solubilization capacity for drugs (2). As a result, utilization of biorelevant media in dissolution testing has increased at least tenfold (as judged by the number of publications) over the last ten years.

The conventional preparation method of these fluids (3) is time-consuming, requires organic solvents, makes daily preparation necessary, and sometimes does not result in a usable medium. Additionally, in conventionally prepared media, residual organic solvents may impact physicochemical properties and dissolution behavior. Elaborate preparation and the high direct costs of the solubilizing agents are reasons why biorelevant media are not used for routine quality control. In earlier studies (4), replacement of the solubilizing excipients with less expensive substitutes was attempted. However, differences in dissolution led to these attempts being abandoned.

Easier and faster preparation combined with high reproducibility and indirect cost savings would make biorelevant media more practical for routine laboratory use. The freeze-drying method enables bulk preparation of storable, instant media. Like the novel, commercially

${ }^{3}$ Corresponding author. available SIF Powder for preparation of FaSSIF and FeSSIF, it can be easily reconstituted before use (5). Media containing the different bile salts were prepared by the conventional method, the freeze-drying method, and the commercially available media. The physicochemical properties, compound solubility, and dissolution behavior of these media were compared.

\section{MATERIALS AND METHODS}

To prepare media by the conventional and the freezedrying methods, pure phosphatidylcholine (PC) from egg and pure sodium taurocholate (NaTC) were used. PC was donated by Lipoid, Germany. NaTC was purchased from PCA, Italy and CPC, New Zealand. SIF Powder instant biorelevant media was purchased from Phares AG, Switzerland. Analytical grade acetonitrile, ammonium acetate, ethanol, isopropanol, methylene chloride, monosodium phosphate, sodium acetate, and sodium hydroxide and HPLC grade methanol were used to prepare the media by different methods or to run analytical methods.

\section{Drugs and Dosage Forms}

A multiparticulate BIXX formulation was provided by Boehringer-Ingelheim Pharma GmbH \& Co. KG (Germany). It is a weakly basic BCS Class II drug with solubility decreasing from $0.082 \mathrm{mg} / \mathrm{mL}$ at $\mathrm{pH} 4.0$ to $0.001 \mathrm{mg} / \mathrm{mL}$ at $\mathrm{pH}$ 6.5. The formulation is not yet commercially available and is therefore referred to as BIXX-F (6).

Danatrol capsules $(100 \mu \mathrm{g})$ were purchased from Sanofi-Synthelabo, Switzerland. This steroidal BCS Class II drug is a neutral and lipophilic compound ( $\log P=4.2$ ) 
that is practically insoluble in water $(1 \mu \mathrm{g} / \mathrm{mL})$. Because danazol is not ionizable, solubility is not pH dependent (7).

\section{Media}

FaSSIF and FeSSIF blank buffers were used for the preparation of the various media. The $\mathrm{pH}$ of FaSSIF blank (0.029 M phosphate, $0.106 \mathrm{M} \mathrm{NaCl}$ ) was adjusted to 6.5 and FeSSIF blank (0.144 M acetate, $0.203 \mathrm{M} \mathrm{NaCl}$ ) was adjusted to $\mathrm{pH}$ 5.0. The method introduced by Galia et al. (2) was used to prepare FaSSIF-CM (CM:conventional method) and FeSSIF-CM. To prepare FaSSIF-FD (FD: freezedried method) and FeSSIF-FD, concentrated solutions of NaTC (from PCA or CPC) and PC in water were freeze-dried, and $2.24 \mathrm{~g} / \mathrm{L}$ or $11.2 \mathrm{~g} / \mathrm{L}$ of lyophilizate were reconstituted in the corresponding blank buffers to prepare the media. Likewise, $2.24 \mathrm{~g} / \mathrm{L}$ and $11.2 \mathrm{~g} / \mathrm{L}$ of instant SIF Powder were dissolved in the corresponding blank buffers to prepare FaSSIF-SIF and FeSSIF-SIF, respectively.

\section{Manufacture of FD Media}

The FD media were prepared by freeze-drying concentrated solutions of biorelevant media, omitting the buffer salts and $\mathrm{NaCl}$. The composition of the media is given in Table 1.

For the preparation of the media, concentrated solutions of all solutes in water were prepared at room temperature for subsequent freeze-drying. To prepare FaSSIF and FeSSIF instant media, a solution containing $247.5 \mathrm{~g} / \mathrm{L}$ sodium taurocholate and $88.5 \mathrm{~g} / \mathrm{L}$ phosphatidylcholine in water was used. The water-soluble taurocholate was dissolved first, followed by the phosphatidylcholine, which was dispersed and subsequently solubilized by the taurocholate, and a clear solution was obtained.

Materials can be dried in bulk or in predosed packages. For bulk drying, a thin layer of solution $(1-2 \mathrm{~cm})$ was poured on a tray. For predosed packaging, the density of the solution was measured to dispense the solution volumetrically into glass vials. The solutions were cooled to $-40{ }^{\circ} \mathrm{C}$ at $1 \mathrm{~K} / \mathrm{min}$. Primary and secondary drying strongly depended on the degree to which the trays or vials were filled and on the freeze-dryer capacity. After freeze-drying, the highly hygroscopic powder had to be handled under dry room conditions or sealed in vials

Table 1. Composition of Concentrates and Freeze-Dried Media per 1-L Vial

\begin{tabular}{lcc}
\hline Substance & $\begin{array}{c}\text { FaSSIF } \\
\text { g in one liter of final } \\
\text { media }\end{array}$ & $\begin{array}{c}\text { FeSSIF } \\
\mathbf{g} \text { in one liter of final } \\
\text { media }\end{array}$ \\
\hline Sodium taurocholate & 1.65 & 8.25 \\
\hline $\mathrm{H}_{2} \mathrm{O}$ & 10 & 35 \\
\hline Phosphatidylcholine & 0.59 & 2.954 \\
\hline Total (dried) & $\mathbf{2 . 2 4}$ & $\mathbf{1 1 . 2 0 4}$ \\
\hline
\end{tabular}

directly under a nitrogen atmosphere. The shelf life of the instant media under these conditions is expected to be 2-3 years at room temperature.

Instant biorelevant media prepared by freeze-drying were white to slightly yellow dry powders that dissolved rapidly in water or aqueous buffer solutions.

Reconstitution of the media was performed with the corresponding "blank" buffers immediately before use.

\section{Characterization of Physicochemical Properties}

The physicochemical properties of the various reconstituted dissolution media were characterized. Surface tension was determined using a bubble-pressure tensiometer (DynoTester, SITA Messtechnik GmbH, Dresden, Germany). Particle-size distribution (PCS) was performed using a Zetasizer Nano_S, Malvern Instruments Limited, Malvern, UK. Density was determined at $20 \pm 2{ }^{\circ} \mathrm{C}$ using a harmonic-oscillation densitometer (DMA 4500, Anton Paar, Graz, Switzerland), and osmolality using a freezing-point osmometer (Osmomat 030-D, Gonotec GmbH, Berlin, Germany). The solubility of danazol (via HPLC-UV-vis) was measured to compare the solubilization capacity of the various media. Danazol $(200 \mu \mathrm{g})$ was weighed into $2-\mathrm{mL}$ Eppendorf tubes and shaken at $400 \mathrm{rpm}, 37^{\circ} \mathrm{C}$ for $24 \mathrm{~h}$. The solutions were filtered using $0.2-\mu \mathrm{m}$ membrane filters (Acrodisc, Pall Life Science, Ann Arbor, USA). The filtrate was diluted 1:1 with $\mathrm{MeOH}$ and measured via HPLC.

\section{Dissolution Tests}

Dissolution was conducted in all biorelevant media and their corresponding blank buffers. In vitro dissolution data were generated at $37 \pm 0.5^{\circ} \mathrm{C}$ in $500 \mathrm{~mL}$ dissolution medium using USP Apparatus 2 (AT 7, Sotax, Allschwill, Switzerland) with the paddle rotating at $75 \mathrm{rpm}$. Samples were removed automatically using a CY-7-50 piston pump (Sotax, Allschwill, Switzerland), filtered online using a GF/D 2.7- $\mu \mathrm{m}$ filter (Whatman, Kent, UK), and then measured online using a UV-vis spectrophotometer (Agilent 8453, Bensheim, Germany). Standard curves for danazol and $\mathrm{BIXX}$ were run every analytical day. The $\mathrm{pH}$ was measured after dissolution of BIXX using a standard $\mathrm{pH}$ meter (CG840, Schott, Hofheim/Ts., Germany) fitted with Inolab electrodes from Mettler Toledo (Schwerzenbach, Switzerland). All dissolution experiments were run in triplicate.

To check the accuracy of the online UV-vis measurements, additional samples were removed manually at predetermined time points and analyzed by HPLC, and the results were compared with the online UV-vis results. These verification samples were filtered through $0.45-\mu \mathrm{m}$ Sartorius membrane filters (Minisart, Sartorius, Goettingen, Germany) and subsequently diluted 1:1 with methanol. Analyses were carried out by HPLC with UV-vis measurement (Agilent HPLC1100, Bensheim, Germany) using a reversed phase column (Zorbax $C_{18}$ Extended Release, Agilent, Bensheim, Germany) and pure water/acetonitrile 
(68/32) as mobile phase for danazol and $0.05 \mathrm{M}$ ammonium acetate $\mathrm{pH} 4.4$ and acetonitrile as mobile phase for BIXX.

\section{RESULTS}

All solutions prepared for freeze-drying appeared optically clear due to the formation of stable, isotropic mixed micelles (8).

\section{Physicochemical Characterization}

Physicochemical properties of media prepared by either reconstitution of freeze-dried media or the conventional method showed no significant differences in surface tension, osmolality, or density (Table 2).

Results obtained from particle-size measurements are given in Table 3. Average diameter $\left(Z_{\text {ave }}\right)$ values of FeSSIF did not show significant differences, with a mean value of $6.4 \mathrm{~nm}$. However, for conventionally prepared FeSSIF, a broad and bimodal distribution with a second peak at 200 $\mathrm{nm}$ was observed, leading to an increased $Z_{\text {ave }}$ of $8 \mathrm{~nm}$. In contrast, a narrow and unimodal distribution was obtained for instant FeSSIF-SIF and freeze-dried FeSSIF-FD.

The particle size of FaSSIF showed higher variance. A stable particle size of about $90 \mathrm{~nm}$ was obtained for freeze-dried FaSSIF. A polydispersity index (PdI) less than 0.05 indicated a narrow, unimodal distribution. The particle size in FaSSIF-SIF is only half that of reconstituted FaSSIF-FD but also shows a narrow, monomodal distribution. We speculate that the smaller particle size might be due to use of soy (S-PC) rather than egg (E-PC) phosphatidylcholine; the different fatty acid composition and higher concentration of unsaturated acids would allow a more compact alignment within the mixed micelles. FaSSIF prepared by the conventional method shows a bimodal distribution with a $Z_{\text {ave }}$ of $88 \mathrm{~nm}$; however, the particles are not stable. Directly after preparation, a $Z_{\text {ave }}$ of about $60 \mathrm{~nm}$ was calculated with a second peak at about $200 \mathrm{~nm}$. Particle size increased to a $Z_{\text {ave }}$ of $90 \mathrm{~nm}$ within one day with a reduction in the intensity of the $200 \mathrm{~nm}$ peak. It can be assumed that equilibrium was achieved at a particle size of $90 \mathrm{~nm}$ in micelles using E-PC and $45 \mathrm{~nm}$ using S-PC. The slow approach to equilibrium particle size makes conventional FaSSIF less reproducible. At least in part, the

Table 2. Physicochemical Properties of FaSSIF and FeSSIF Prepared by Reconstitution of Freeze-Dried Media or by the Conventional Method

\begin{tabular}{lcc}
\hline Property & FaSSIF & FeSSIF \\
\hline Surface tension $(\mathrm{mN} / \mathrm{m})$ & $53 \pm 0.4$ & $47.4 \pm 0.3$ \\
\hline Osmolality $(\mathrm{mosmol} / \mathrm{kg})$ & $1.00693 \pm 0.00004$ & $1.01484 \pm 0.00015$ \\
\hline Density $\left(\mathrm{mg} / \mathrm{cm}^{3}\right)$ & $296 \pm 3$ & $706 \pm 9$ \\
\hline
\end{tabular}

\begin{tabular}{|c|c|c|c|c|}
\hline Medium & $\mathrm{Z}_{\text {ave }}^{\text {mean }}(\mathrm{nm})$ & SD Z & mean Pdl & SD PdI \\
\hline FaSSIF FD CPC ${ }^{a}$ & 89 & 2 & 0.046 & 0.015 \\
\hline FaSSIF FD PCA ${ }^{b}$ & 89 & 1 & 0.038 & 0.012 \\
\hline FaSSIF CM CPC & 88 & 11 & 0.124 & 0.019 \\
\hline FaSSIF CM PCA ${ }^{d}$ & 88 & 15 & 0.038 & 0.012 \\
\hline FaSSIF-SIF & 45 & 2 & 0.025 & 0.008 \\
\hline FeSSIF FD CPC & 6.4 & 0.1 & 0.071 & 0.013 \\
\hline FeSSIF FD PCA & 6.4 & 0.3 & 0.130 & 0.066 \\
\hline FeSSIF CM CPC & 8 (6 and 200) & 0.4 & 0.404 & 0.071 \\
\hline FeSSIF-SIF & 6.4 & 0.1 & 0.090 & 0.015 \\
\hline
\end{tabular}

${ }^{a}$ Bile salts from $C P C$, New Zealand

${ }^{b}$ Bile salts from PCA, Italy

' $2 \mathrm{~h}$ after preparation

${ }^{d} 24 \mathrm{~h}$ after preparation

high standard deviations in solubility and physicochemical parameters with conventional FaSSIF can be explained by the variation in the rate of approach to equilibrium in the test solutions. Using instant SIF Powder or freeze-dried media, equilibrium was reached immediately after reconstitution.

The solubility of danazol in FaSSIF and FeSSIF was 15 and $49 \mu \mathrm{g} / \mathrm{mL}$, respectively, in conventionally prepared media. In the freeze-dried media, 14 and $48 \mu \mathrm{g} / \mathrm{mL}$ could be dissolved. Use of bile salts from different suppliers (PCA vs $(P C)$ had no impact on the results. The very slightly higher concentrations in conventionally prepared media may have resulted from residual organic solvent, which could cause increased solubility of danazol. In the SIF Powder media, the solubility of danazol was marginally lower, with values of 13 and $47 \mu \mathrm{g} / \mathrm{mL}$. If soy lecithin was indeed used to manufacture this preparation, the tighter mixed micelles formed might show a slightly reduced capacity for solubilizing additional molecules. However, for all solubility measurements, no significant differences were calculated with either the $F$ or $t$-tests.

\section{Dissolution Results \\ Danazol}

Figure 1 shows the mean dissolution of danazol from Danatrol capsules. The profile obtained in blank buffers is not included because the solubility of danazol is less than $1 \%$ in the blank solutions.

Dissolution of danazol from Danatrol capsules in FeSSIF was about threefold higher than in FaSSIF. In blank buffers, no difference could be observed, indicating that the effect on solubility is dependent on surfactant levels 


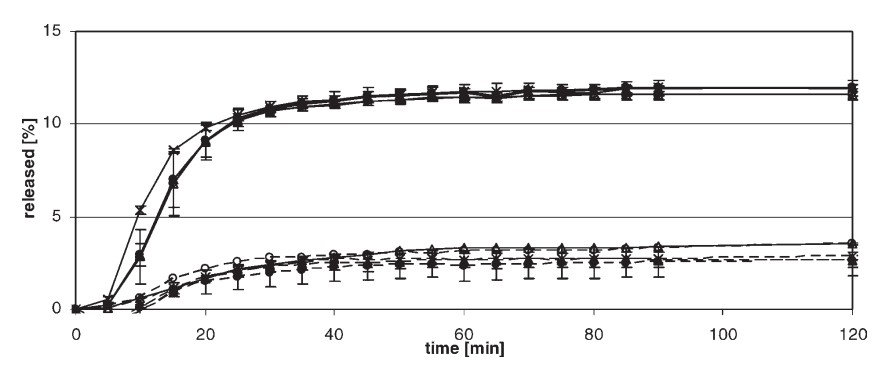

Figure 1. Mean dissolution values and standard deviations for danazol in FaSSIF (- ) and FeSSIF (-):SIF (x), FD CPC ( $\mathbf{\Delta}), \operatorname{FD} P C A(\Delta), C M C P C(\mathbf{0})$, and CMPCA (O).

and not on $\mathrm{pH}$. For FaSSIF, no significant difference in dissolution profiles could be calculated using two-way ANOVA for repeated measurements among the various media-preparation methods. The dissolution in FeSSIF showed no significant difference from that in the freezedried and conventionally prepared media. FeSSIF prepared using instant SIF Powder showed a significantly higher percentage release at time points between 10 and 20 min using the post hoc Bonferroni test.

\section{BIXX}

Figure 2 shows the mean dissolution of BIXX from the formulation in FaSSIF. The source (supplier) of bile salt had little effect on the results. The maximum concentration in conventionally prepared media was reached in $35 \mathrm{~min}$, whereas in the FD and SIF FaSSIF, the maximum concentration was reached after $40 \mathrm{~min}$. The mean maximum concentrations were $15.3 \%$ released in conventionally prepared media, 16\% in FD, and 18\% in FaSSIF-SIF. None of these differences were statistically significant. In all FaSSIF media, an initially higher percentage released reverted to a final value of $7.5 \pm 0.5 \%$ released by $120 \mathrm{~min}$. The high initial solubility is driven by excipients, like supersaturating agents or $\mathrm{pH}$ modifiers, that effect supersaturation and cause a delay in re-precipitation of the API, whereas the final percentage released is defined by the saturation solubility.

Figure 3 shows the mean dissolution data for the BIXX formulations in the various FeSSIF media. The stable $\mathrm{pH}$ generated by the FeSSIF buffers led to a final release of just $2 \%$ in the blank media. The strong solubilizing effect in FeSSIF led to a threefold higher percentage released with dissolution continuing over an extended period. A final percentage release of $40 \%$ was reached after $4 \mathrm{~h}$ (data not shown).

Acidifying excipients in the formulation can cause a decrease in $\mathrm{pH}$, depending on the buffer capacity of the medium, which in turn affects the solubility of the weakly basic BIXX compound. However, such effects depend on the buffer capacity of the dissolution medium. A pH drift from 6.5 to 6.14 was observed in FaSSIF, which has a low buffer capacity, whereas in FeSSIF, the $\mathrm{pH}$ remained stable at 5.0 .

Dissolution Technologies | AUGUST 2009

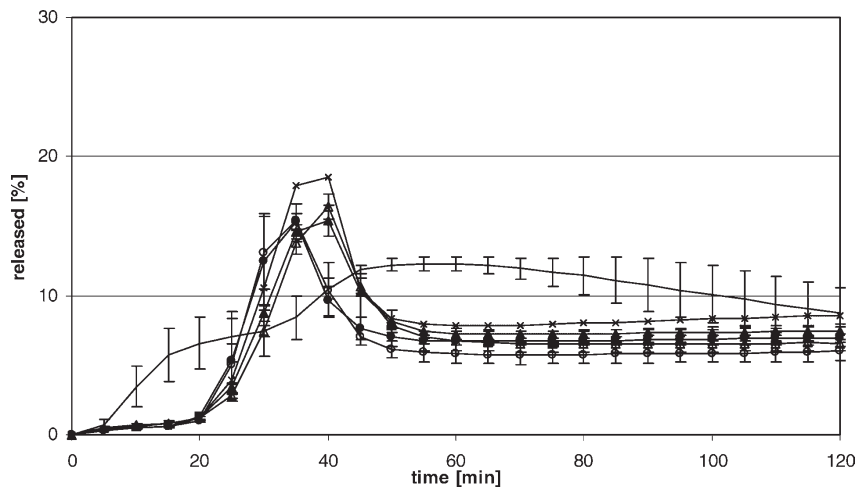

Figure 2. Mean dissolution values and standard deviations for BIXX in FaSSIF: $\operatorname{SIF}(x), F D C P C(\mathbf{\Delta}), F D P C A(\Delta), C M C P C(\bigcirc), C M P C A(\bigcirc)$, and buffer blank (without symbol).

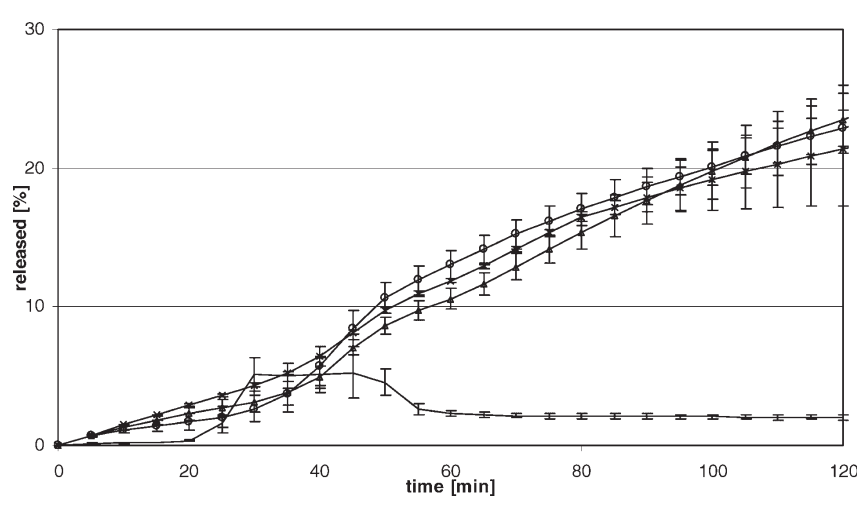

Figure 3. Mean dissolution values and standard deviations for BIXX in FeSSIF: $\operatorname{SIF}(x), F D C P C(\mathbf{\Delta}), F D P C A(\Delta), C M C P C(\bullet)$, and buffer blank (without symbol).

\section{CONCLUSIONS \\ Effect of Sodium Taurocholate (NaTC) Source on Media Performance}

Results with the sodium taurocholate from CPC with respect to the physicochemical properties and solubilizing effects of the biorelevant media as well as the dissolution behavior of danazol and BIXX were equivalent to those obtained with the product from PCA. Therefore, substitution of NaTC from CPC for the more expensive NaTC from PCA can be recommended.

\section{Comparison of Freeze-Dried Media versus Conventional Preparation Method}

Media reconstituted from the freeze-dried product showed very similar properties but higher reproducibility than freshly prepared media. The freeze-dried FaSSIF and FeSSIF can be manufactured on a larger scale to produce material to supply the media for several weeks or even months, depending on the equipment used. Furthermore, reconstitution of the freeze-dried FaSSIF and FeSSIF is facile, so the problems sometimes encountered with manufacture of the freshly prepared media are 
circumvented. For both reasons, savings in costs of the taurocholate and lecithin and in the time required for preparation in the laboratory can be realized. The freezedried media, therefore, have substantial advantages over the freshly prepared media.

\section{SIF Powder versus Freeze-Dried FaSSIF and FeSSIF}

The SIF Powder media, which are speculated to contain soy phosphatidylcholine instead of egg phosphatidylcholine, lead to a slightly different particle size of the mixed micelles. However, the solubilization capacity of SIF Powder-based media for danazol and the dissolution of BIXX were similar for all media. Even though the dissolution of danazol in FeSSIF was initially slightly faster in media prepared from SIF Powder, the maximum concentration and the time at which this was reached were identical. Therefore, either of the "instant" media (freeze-dried or SIF Powder) would be suitable for substitution of the freshly prepared media.

\section{REFERENCES}

1. Dressman, J. B.; Amidon, G. L.; Reppas, C.; Shah, V.P. Dissolution testing as a prognostic tool for oral drug absorption: immediate release dosage forms. Pharm. Res. 1998, 15, 11-22.

2. Galia, E.; Nicolaides, E.; Horter, D.; Lobenberg, R.; Reppas, C.; Dressman, J. B. Evaluation of various dissolution media for predicting in vivo performance of class I and II drugs. Pharm. Res. 1998, 15, 698-705.
3. Marques, M. Dissolution media simulating fasted and fed states. Dissolution Technol. 2004, 11, 16.

4. Vertzoni, M.; Fotaki, N.; Kostewicz, E.; Stippler, E.; Leuner, C.; Nicolaides, E.; Dressman, J. B.; Reppas, C. Dissolution media simulating the intralumenal composition of the small intestine: physiological issues and practical aspects. J. Pharm. Pharmacol. 2004, 56, 453-462.

5. Quinton, J.; Streich, D.; Geissbuehler, R.; Schwebel, H.; van Hoogevest, P. M.; Basit, A.; Leigh, M.; Grunkemeyer, J. Instantly Dissolving Powders for Producing Standardized Biorelevant Dissolution Media for Quality Control of Poorly Water Soluble Drugs. AAPS Annual Meeting and Exposition, San Antonio, TX, Oct 29-Nov 2, 2006.

6. Wunderlich, M. Biorelevante in vitro Methoden zur Vorhersage des in vivo Verhaltens von schlecht wasserlöslichen, schwach basischen Arzneistoffen. Johann Wolfgang Goethe Universität: Frankfurt am Main, Germany, 2004.

7. Pedersen, B. L.; Brondsted, H.; Lennernas, H.; Christensen, F. N.; Mullertz, A.; Kristensen, H. G. Dissolution of hydrocortisone in human and simulated intestinal fluids. Pharm. Res. 2000, 17, 183-189.

8. Lichtenberg, D.; Robson, R. J.; Dennis, E. A. Solubilization of phospholipids by detergents. Structural and kinetic aspects. Biochim. Biophys. Acta 1983, 737, 285-304.

\section{A. Gray Consulting, lnc. On Site Dissolution Traiming and Lab Inspection Setvices}

Dissolution expert Vivian Gray of V. A. Gray Consulting, Inc., now offers an on-site service that provides:

+ Training of personnel in dissolution testing.

* An inspection of the lab that runs the dissolution tests to alert analysts and management of any shortcomings relative to GMP requirements and general industry practice.

* An evaluation of equipment testing and qualification protocols.

* Instruction on general lab techniques and calibration aspects (chemical and mechanical) with hands-on testing.

* A training seminar tailor-made to the functions of the labs involved (e.g., Quality Control or Research and Development).

Training certificates.

Seminar course material on CD.

For more information, contact Vivian Gray at vagray@rcn.com and visit www.vagrayconsulting.net 